\title{
Numerical interpolation methods applied in electromagnetic interference problems
}

\author{
Dan D. Micu ${ }^{1}$, Andrei Ceclan ${ }^{1}$, Iosif Lingvay ${ }^{2}$, and Emil Simion ${ }^{1}$ \\ ${ }^{1}$ Department of Electrical Engineering, Technical University of Cluj-Napoca \\ Baritiu str. 24-26, 400020 Cluj-Napoca (Romania) \\ Phone/Fax number:+0040 264401462, e-mail: Dan.Micu@et.utcluj.ro \\ ${ }^{2}$ INCDIE ICPE-Cercetări Avansate, ICPE SA Bucuresti, (Romania) \\ e-mail: coroziune@icpe-ca.ro
}

\begin{abstract}
In the paper are evaluated the induced potential in pipeline based on the subdivision of the zone of influence AC Power Lines / electric traction line / gas pipeline, in a relatively great number of sections in order to be able to determine voltages at many positions along the gas pipeline. Is presented a practical case from Cluj, of subdivision of the zone of influence in sections (a circuit model) and is created a special interpolation algorithm for the precise calculation of the induced voltages values in different points on the pipeline taking into account the measured data.

Because there are only a set of available reactance measurements, for a continuous function between the magnetic permeability and electrical induced currents, it is necessary to apply numerical interpolation methods and/or nonlinear curve fitting procedures.
\end{abstract}

\section{Key words}

Induced voltage, electromagnetic interference, interpolation algorithm, power systems.

\section{Introduction}

Pipelines located near power lines and electric traction lines, may capture a portion of the energy encompassed by the conductors' paths, particularly under unfavourable circumstances such as long parallel exposures and power fault conditions [1], [2].

Interference calculations consist essentially of inductive and conductive interference calculations, which are performed independently; computation results can subsequently be combined together, see figure 1 .

Thus it is seen that inductive interference calculations are performed on a hybrid field theory/circuit theory model.

Conductive interference calculations deal with buried conductors exclusively.

Conductors are first subdivided into segments of lengths small enough with respect to both wavelength and overall length of the ground network such that they will lead satisfactory to the desired engineering accuracy.

In the figure 2 are presented a corrosion case of a gas pipeline due to the a.c. and d.c. dispersion currents. [3]

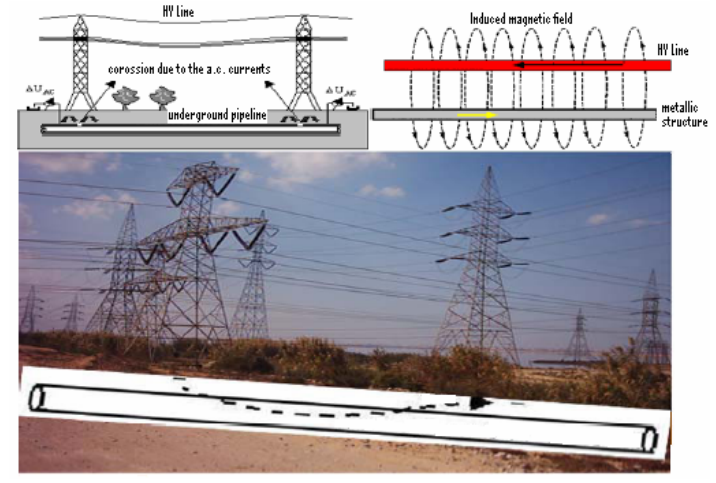

Fig. 1. Complex right - of - way configuration

A finite elements field theory approach is then used to relate the currents and potentials in the segments in such a way that an equivalent circuit model can be created which involves the internal and external impedances of the segments [1], [2].

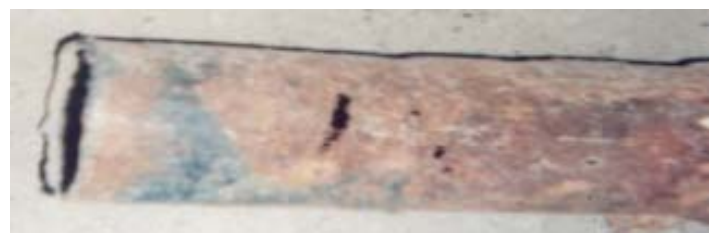

Fig. 2. Corrosion of a gas pipeline due to the a.c. and d.c. dispersion currents

Hence circuit theory can be applied once again to obtained potentials at all segment end points, as well as longitudinal and leakage currents in every conductor segment in a quite straightforward manner.

\section{Mathematical evaluation of the induced voltages}

Calculation of the voltages appearing on the pipelines is work out in two steps. The first step is the determination of the electromotive forces (EMF) induced along the pipeline (EMF cause currents circulation in the pipeline and voltages between the pipeline and surrounding earthis a longitudinal electromotive force induced by 
transmission line current) and the second step is to calculate the voltages to earth and the circulating current in response to the induced longitudinal electromotive forces. See in figure 3 the equivalent circuit formed by the pipeline and the earth [2].

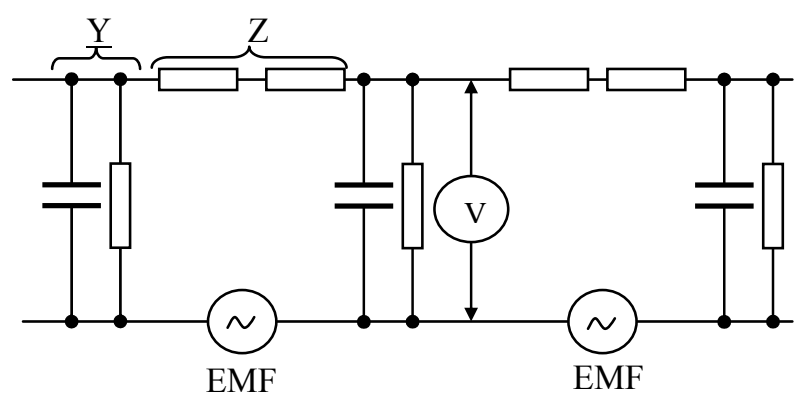

Fig. 3. Equivalent circuit pipeline - soil

A clear distinction has to be made between EMF and voltages appearing on the pipeline.

EMF's are virtually electric generators inside the circuit pipeline/earth resulting from the influence of the magnetic coupling.

These EMF's produce voltages on the pipeline, and only these voltages $\mathrm{V}$ represent the actual stresses on the pipeline and its equipment. The evaluation is based on the subdivision of the zone of influence in a relatively great number of sections [1], [2].

This is done in order to be able to determine voltages at many positions along the pipeline.

Each section is represented by a cell submitted to the influence of the electromotive force calculated according the method developed for normal operation (steady state condition) and fault conditions [4].

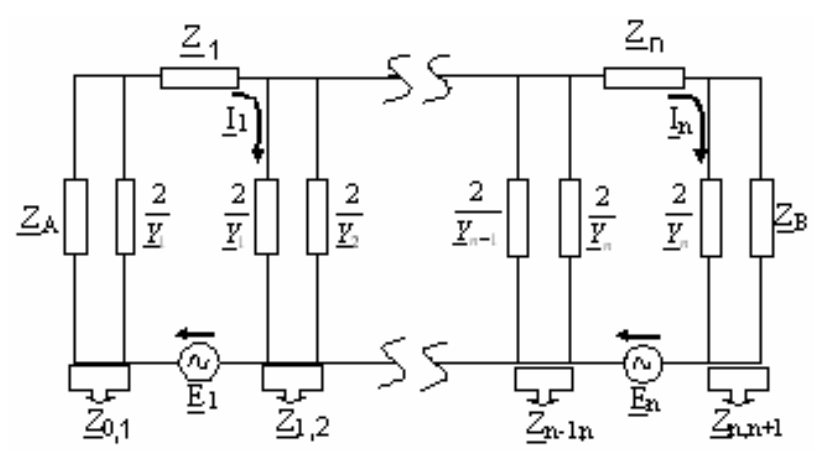

Fig. 4. Equivalent circuit $-\pi$ cell

Except for the voltage source, figure 5 present an equivalent elemental pipeline circuit which is identical to the elemental circuit for the usual electrical transmission line with the same definitions of series impedance per unit length $\underline{Z}=R+j \omega L$, and shunt admittance per unit length, $\underline{Y}=C+j \omega C$.

Using the electrical transmission line parameters is determined the induced potential in pipeline:

Where

$$
V(x)=Z_{C}\left\{\left[K_{1}+P(x)\right] e^{-\gamma x}-\left[K_{2}+Q(x)\right] e^{\gamma x}\right\}
$$

$$
\begin{aligned}
& P(x)=\frac{1}{2 Z_{C}} \int_{x_{A}}^{x} e^{\gamma s} E_{x}(s) d s \\
& Q(x)=\frac{1}{2 Z_{C}} \int_{x}^{x_{B}} e^{-\gamma s} E_{x}(s) d s
\end{aligned}
$$

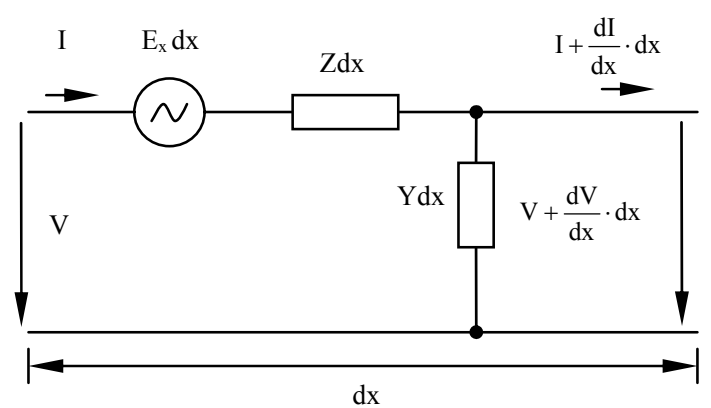

Fig. 5. Equivalent elemental pipeline circuit

and the coefficients resulting for the boundary conditions at ends:

$$
\begin{gathered}
K_{1}=v_{1} e^{\gamma x_{A}} \cdot \frac{v_{2} P\left(x_{B}\right) e^{-\gamma x_{B}}-Q\left(x_{A}\right) e^{\gamma x_{B}}}{e^{\gamma\left(x_{B}-x_{A}\right)}-v_{1} v_{2} e^{-\gamma\left(x_{B}-x_{A}\right)}} \\
K_{2}=v_{2} e^{-\gamma x_{B}} \frac{v_{1} Q\left(x_{A}\right) e^{\gamma x_{A}}-P\left(x_{B}\right) e^{-\gamma x_{A}}}{e^{\gamma\left(x_{B}-x_{A}\right)}-v_{1} v_{2} e^{-\gamma\left(x_{B}-x_{A}\right)}}
\end{gathered}
$$

For the induced potential evaluation is used an approximation which consists in a uniform solicitation on the length of the influence zone:

$$
E_{0}=\frac{\sum_{i=1}^{n} E_{i} \cdot L_{i}}{L_{t}}=\frac{\sum_{i=1}^{n} E_{i} \cdot L_{i}}{\sum_{i=1}^{n} L_{i}}
$$

where $E_{i}$ is the electromotive force on the zone with length $L_{i}$ and $L_{t}$ is the length of the entire influence zone electric line-pipeline [4].

At the extremity $\mathrm{L}_{1}$, the influence of the line is equivalent with a voltage source with a value equal to the value of the voltage obtained with the following formula [5]:

$$
\underline{V}(x)=\frac{E_{0}}{\gamma} \frac{\underline{Z}_{A} \underline{Z}_{B}\left[\operatorname{ch}(\gamma x)-\operatorname{ch}\left(L_{4}-x\right)\right]+\underline{Z}_{C}\left[\underline{Z}_{B} \operatorname{sh}(\gamma x)+\underline{Z}_{A} \operatorname{sh}\left(L_{4}-x\right)\right]}{\left(\underline{Z}_{A} \underline{Z}_{B}+\underline{Z}_{C}^{2}\right) \operatorname{sh}\left(\gamma_{4}\right)+\underline{Z}_{C}\left(\underline{Z}_{A}+\underline{Z}_{B}\right) \operatorname{ch}\left(\gamma_{4}\right)}
$$

For $\underline{Z}_{B}=\infty$ and $\underline{Z}_{A}=\underline{Z}_{0}$ is obtained:

$\underline{V}_{B}=E_{1} \cdot \frac{\underline{Z}_{A}\left(\operatorname{ch} \gamma L_{1}-1\right)+\underline{Z}_{0} \operatorname{sh} \gamma L_{1}}{\gamma \cdot\left(\underline{Z}_{A} \operatorname{sh} \gamma L_{1}+\underline{Z}_{0} \operatorname{ch} \gamma L_{1}\right)}=-\frac{E_{1}}{\gamma}\left(1-e^{-\gamma L_{1}}\right)$

and the source impedance:

$$
\underline{Z}_{B}=\frac{\underline{Z}_{0} \operatorname{sh}\left(\gamma L_{1}\right)+\underline{Z}_{A} \operatorname{ch}\left(\gamma L_{1}\right)}{\underline{Z}_{0} \operatorname{ch}\left(\gamma L_{1}\right)+\underline{Z}_{A} \operatorname{sh}\left(\gamma L_{1}\right)}
$$


The $\underline{Z}_{\mathrm{A}}$ impedance is the circuit pipeline/soil impedance located at the left side of the A point.

At the extremity $L_{2}$, with an electromotive force $E_{2}$, the equivalent voltage source is the sum of the sources given by the influence of $\mathrm{AB}$ and $\mathrm{BC}$ zone [4]:

$$
\begin{gathered}
V_{C}=V_{B} \cdot e^{-\gamma L_{2}}+V_{C}^{*} \\
V_{C}^{*}=E_{2} \cdot \frac{Z_{B}\left(c h \gamma L_{2}-1\right)+Z_{0} \operatorname{sh} \gamma L_{2}}{\gamma \cdot\left(Z_{B} \operatorname{sh} \gamma L_{2}+Z_{0} \operatorname{ch} \gamma L_{2}\right)}=-\frac{E_{2}}{\gamma}\left(1-e^{-\gamma L_{2}}\right)
\end{gathered}
$$

with: $\underline{Z}_{B}=\underline{Z}_{0} ; \underline{Z}_{A}=\underline{Z}_{B}=\underline{Z}_{0}$, so:

$$
\underline{V}_{C}=\frac{E_{1}}{\gamma}\left(1-e^{\gamma L_{1}}\right) e^{-\gamma L_{2}}+\frac{E_{2}}{\gamma}\left(1-e^{-\gamma L_{2}}\right)
$$

\section{Electromagnetic interference study}

Some authors [12] approach the electromagnetic interference between power grids and neighbouring metalic structures, by proposing the construction of a database to contain the corresponding values of the relative magnetic permeabilities of the metalic parts and the values of the induced electrical currents on different frequencies, both in a normal function of the power grids, and in mallfunction conditions.

Thus, a reconsideration of the relative magnetic permeability, in an approximation close to the reality, leads also to relevant estimations of the electrical interference values that are to be identified.

An elocquent example on this issue may be explained by one of the mathematical relations which relates the conductor imepdances with the magnetic permeabilities, by Carson theory.

Because there are only a set of available reactance measurements, for a continuous function between the magnetic permeability and electrical induced currents, it is necessary to apply numerical interpolation methods and/or nonlinear curve fitting procedures.

Using numerical data from the consulted bibliography [5], [6], [7], [8] we implemented and tested a set of numerical interpolation instruments. Special attention was paid to the minimisation of the interpolation error, and to the stability of the proposed functions.

The numerical interpolation of the magnetic permeability as related to the variation of the induced electrical current in the metalic structures was accomplished with a range of liniar, Lagrange and spline functions. [11]

The relation below shows the cubic spline models, used in the interpolation process of this study:

$$
B(x)=\left\{\begin{array}{cc}
0 & x \leq-2 \\
(x+2)^{3} & -2 \leq x \leq-1 \\
1+3 \cdot(x+1)+3 \cdot(x+1)^{2}-3 \cdot(x+1)^{3} & -1 \leq x \leq 0 \\
1+3 \cdot(1-x)+3 \cdot(1-x)^{2}-3 \cdot(1-x)^{3} & 0 \leq x \leq 1 \\
(2-x)^{3} & 1 \leq x \leq 2 \\
0 & 2 \leq x
\end{array}\right.
$$

The cubic spline interpolation has to be defined as a liniar combination of the $B_{\mathrm{i}}$ functions in the known interpolation knots.

$$
f\left(x_{k}\right)=\sum_{i=-1}^{n+1} c_{i} \cdot B_{i}\left(x_{k}\right) \quad 0 \leq k \leq n
$$

In order to identify the $c_{\mathrm{i}}$ coefficients from the spline interpolation function, we have to solve a tridiagonal system of equations, that without imposing any boundary conditions to the interpolation function, proves to be highly ill-conditioned. That is, a small variation of the input data can lead to great variations of the output data, the coefficients. Thus, the stability of the interpolation function is greatly affected.

Numerical regularization techniques have to be applied so as to reach stable coefficients of the spline interpolation functions. [9], [10]

The tridiagonal equations system is presented in the above relation:

$$
\left[\begin{array}{ccccc}
1 & 4 & 1 & & \\
& 1 & 4 & 1 & \\
& \cdots & \cdots & \cdots & \\
& & 1 & 4 & 1
\end{array}\right] \cdot\left[\begin{array}{c}
c_{-1} \\
c_{0} \\
\ldots \\
c_{n+1}
\end{array}\right]=\left[\begin{array}{c}
f\left(x_{0}\right) \\
f\left(x_{1}\right) \\
\ldots \\
f\left(x_{n}\right)
\end{array}\right]
$$

As it can be seen in Fig. 6, the interpolation functions, linear and spline, do a great job in fitting a continuos link function between magnetic permeability and induced electrical currents. The cspline function interpolates with cubic functions, lspline approximates the boundary knots with liniar functions, and pspline with parabolic functions.

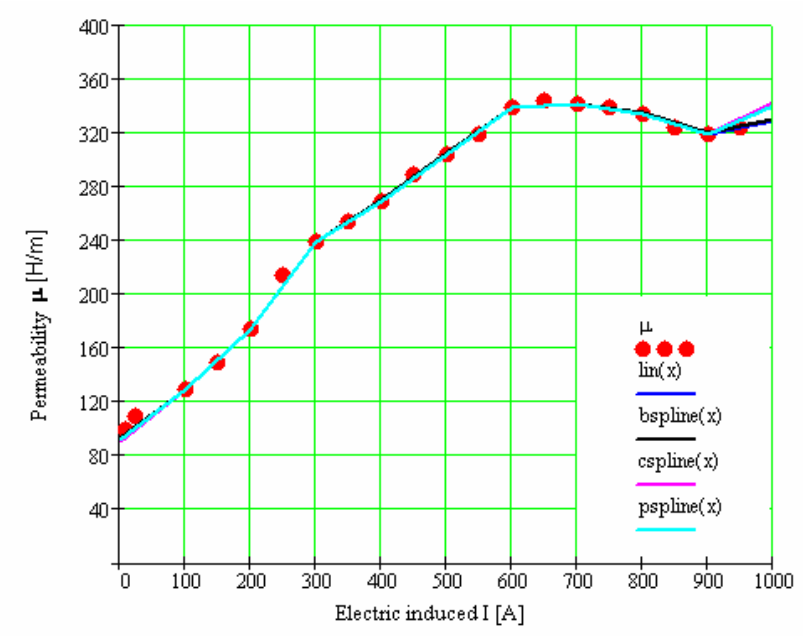

Fig. 6. Numerical interpolation of data aqusition

\section{Induced voltages for a real case}

Is made an evaluation of the induced voltages in a pipeline, which runs in the same right of way with a power line and electric traction line, for a real case, figure 7 [6].

The input data of this problem are: power line and pipelines geometrical configuration; conductor and pipeline physical characteristics (including insulating and coating characteristics); environmental parameters (air characteristics, soil structure and characteristics); power system terminal (or boundary) parameters (power source voltages, equivalent source impedances) [3]. 


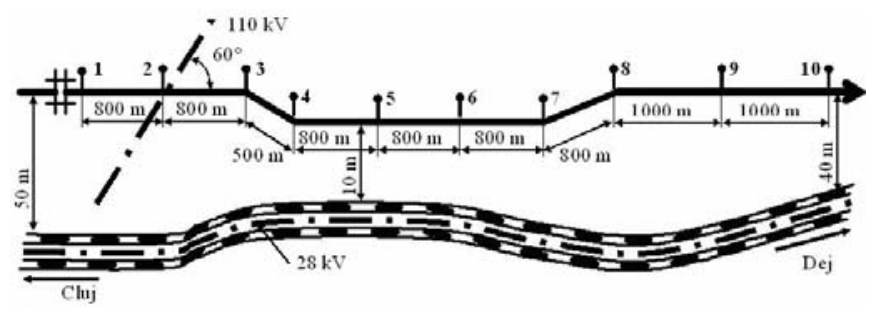

Fig. 7. The right of way electric power line-traction linegas pipeline

The results demonstrates that is possible to obtain a precise evaluation of the solicitations if is known the resistance and the adduction current in the pipeline.

After the determination in each point the potentials due to the right and left side of the line is applied the superposition method [2], [3].

It was measured the a.c. voltages between the pipe and soil, respectively the $\mathrm{U}_{\mathrm{AC}}$ induced along the gas pipeline. We measured the induced alternative voltages in steel gas pipelines posed in different type of soil and isolated by different organic layers. In this way was determined the:

- induced voltages $\mathrm{U}_{\mathrm{CA}}$ in a medium pressure gas pipeline (1999), with 300mm diameter and $8 \mathrm{~mm}$ width, isolated with polyethylene in 3 layers (total $3 \mathrm{~mm}$ ) according with the EN 10285, having the same right of way $(20 \mathrm{~km})$ with a double traction line and being intersected with an electric power line $110 \mathrm{kV}$

- $\quad$ soil resistivity along the utility corridor

- electrochemical potentials E pipeline/soil along the gas pipeline

The results are presented in the table below [6]:

\begin{tabular}{|c|c|c|c|c|c|c|c|c|c|c|}
\hline Measured parametess & \multicolumn{10}{|c|}{ Points of measures } \\
\hline & 1 & 2 & 3 & 4 & 5 & 6 & 7 & 8 & 9 & 10 \\
\hline Soil resistivity $[0 \mathrm{~m}]$ & 11 & 18 & 16 & 45 & 32 & 93 & 21 & 64 & 17 & 15 \\
\hline $\mathrm{U}_{\mathrm{A}}[\mathrm{Vi}]$ & 8,10 & 19,1 & 17,2 & 293 & 15,2 & 23,4 & 7,13 & 1,89 & 2,11 & 4,32 \\
\hline$E\left[\mathrm{~V}_{\text {Cucusou }}\right]$ & $-0,353$ & $-0,398$ & 0,371 & $-0,325$ & $-0,394$ & $-0,410$ & $-0,307$ & 0,324 & $-0,352$ & $-0,365$ \\
\hline
\end{tabular}

Is briefly presented in MathCad, a program using some interpolation predefined functions for the induced voltage calculation in different points on the pipeline, for some measured data. Is observed that the interpolation spline function with linear end conditions is more precise than the polynomial linear interpolation [7], [8].

Is made in MathCad an algorithm which use the predefined interpolation function and in figure 8 is represented the measured values and the interpolation functions for the induced voltages on the commune zone at various distances.

Also, are presented the results obtained with spline and linear interpolation of the measured data. The calculus and the program made in MathCad with the predefined functions help us to determine more precisely the values of the induced voltages on the entire influence zone.

The values in volt of the induced voltages $(\mathrm{y})$ measured on the common corridor line-pipeline at different distances in meters (x), are introduced in MathCad program.

$$
\mathrm{x}:=\left(\begin{array}{c}
800 \\
1600 \\
2400 \\
2650 \\
3450 \\
4250 \\
5050 \\
5450 \\
6450 \\
7450
\end{array}\right) \quad \mathrm{y}:=\left(\begin{array}{c}
8.10 \\
19.10 \\
17.2 \\
29.3 \\
15.2 \\
26.4 \\
7.13 \\
1.89 \\
2.11 \\
4.32
\end{array}\right)
$$

The predefined functions for the spline and linear interpolation are used for the induced voltage calculus in any point of the right of way.

$$
\begin{aligned}
& \mathrm{L}:=\operatorname{lspline}(\mathrm{x}, \mathrm{y}) \quad \mathrm{C}:=\operatorname{cspline}(\mathrm{x}, \mathrm{y}) \quad \mathrm{P}:=\operatorname{pspline}(\mathrm{x}, \mathrm{y}) \\
& \operatorname{lsp}(\mathrm{t}):=\operatorname{interp}(\mathrm{L}, \mathrm{x}, \mathrm{y}, \mathrm{t}) \quad \operatorname{csp}(\mathrm{t}):=\operatorname{interp}(\mathrm{C}, \mathrm{x}, \mathrm{y}, \mathrm{t}) \\
& \operatorname{psp}(\mathrm{t}):=\operatorname{interp}(\mathrm{P}, \mathrm{x}, \mathrm{y}, \mathrm{t}) \quad \mathrm{l}(\mathrm{t}):=\operatorname{linterp}(\mathrm{x}, \mathrm{y}, \mathrm{t})
\end{aligned}
$$

$\mathrm{t}:=800,801 . .7450$

We develop in MathCAD program an algorithm for the induced voltage calculation in different points on the pipeline based on the measured data.

Is observed that the interpolation spline function with linear end conditions is more precise than the linear interpolation polynom but the created algorithm gives us the correct data if the interval of the influence zone is increasing.

$$
\begin{aligned}
& A:=\mid \begin{array}{l}
X \leftarrow \mathrm{X} \\
\mathrm{V} \leftarrow \mathrm{V}
\end{array} \\
& \mathrm{n} \leftarrow \operatorname{last}(\mathrm{X}) \\
& \text { for } \mathrm{j} \in 0 . . \mathrm{n} \\
& \mathrm{A}_{\mathrm{j}, 0} \leftarrow \mathrm{V}_{\mathrm{j}} \\
& \text { for } \mathrm{j} \in 1 . . \mathrm{n} \\
& \mid \begin{array}{l}
\text { for } \mathrm{k} \in 0 . . \mathrm{n}-\mathrm{j} \\
\mathrm{A}_{\mathrm{k}, \mathrm{j}} \leftarrow \frac{\mathrm{A}_{\mathrm{k}+1, \mathrm{j}-1}-\mathrm{A}_{\mathrm{k}, \mathrm{j}-1}}{\mathrm{X}_{\mathrm{k}+\mathrm{j}}-\mathrm{X}_{\mathrm{k}}}
\end{array} \\
& \text { for } \mathrm{i} \in 1 . . \mathrm{j} \\
& A_{n-j+i, j} \leftarrow 0 \\
& \text { A } \\
& \mathrm{j}:=1 . . \text { last }(\mathrm{x}) \quad \text { last }(\mathrm{x})=3 \quad \mathrm{z}:=56,58 . .86 \\
& \mathrm{~N}(\mathrm{z}):=\sum_{\mathrm{j}}\left[\mathrm{A}_{0, \mathrm{j}} \cdot \prod_{\mathrm{i}=0}^{\mathrm{j}-1}\left[\mathrm{z}-(\mathrm{x})_{\mathrm{i}}\right]\right]+\mathrm{A}_{0,0}
\end{aligned}
$$




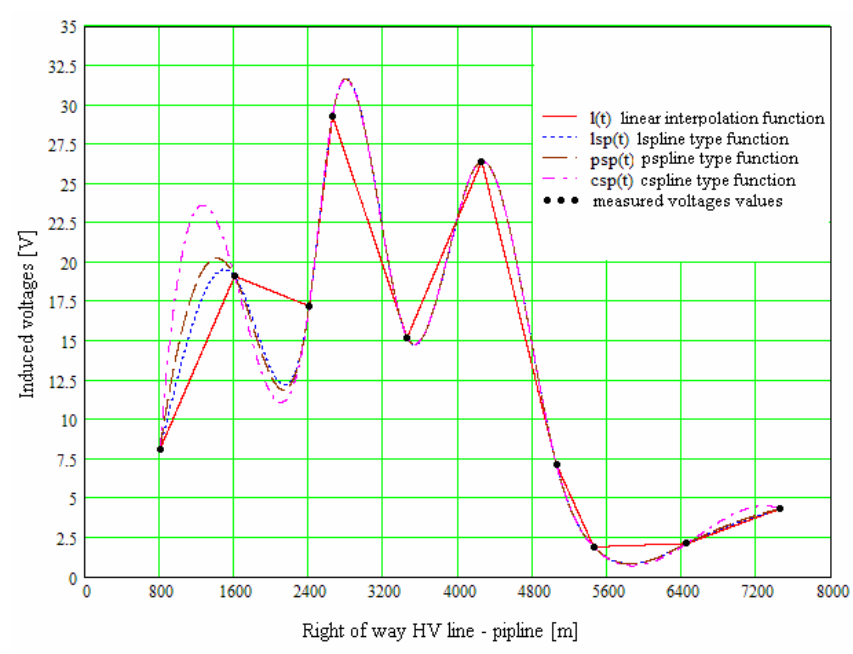

Fig. 8. Induced voltages in the gas pipeline using special interpolation functions

\section{Conclusion}

The evaluation of the induced voltage in pipeline is based on the subdivision of the zone of influence in a relatively great number of sections in order to be able to determine voltages at many positions along the pipeline using Thevenin equivalent circuits. Is presented a practical case of subdivision of the zone of influence in sections (a circuit model) and is made a precise evaluation of the induced voltages using some interpolation functions for the measured data. Is observed that the interpolation spline function with linear end conditions is more precise than the linear interpolation polynom but the presented MathCad algorithm gives us the correct data if the interval of the influence zone is increasing. The interpolation functions, linear and spline, do a great job in fitting a continuos link function between magnetic permeability and induced electrical currents.

\section{Acknowledgement}

The authors are grateful to the Romanian Minister of Scientific Research and Technology for the financial support in the frame of CEEX - ICEMECOS 2006 X2C37 Research Program.

\section{References}

[1] F. Dawalibi, "Analysis of electrical interference from power lines to gas pipelines-PartI-Computation method", PWRD-4, No3, pp. 1840-1848, July 1989.

$[2]^{* * *}$, "Guide Concerning Influence of High Voltage AC Power Systems on Metallic Pipelines", CIGRE Working Group 36.02, Canada, 1995.

[3] D.D. Micu, I. Lingvay, E. Simion, "Modelarea şi predicția fenomenelor de interferență în regim electrocinetic" (in romanian), Ed. ELECTRA, Bucureşti, 2006.

[4] ***, "Resultats des measures et interference sur la ligne Neufchateau/Villers-sur-Semois et la canalization de gaz distrigaz Ben-Ahin/Athus“, Laborelec Brussel, Research Report 1990.
[5] D. D. Micu, E. Simion, A. Ceclan, D. Micu, "Numerical methods for induced voltage evaluation in electromagnetic interference problems", 9 th International Conference EPQU Barcelona, 9-11 Oct. 2007.

[6] D.D. Micu, I. Lingvay, E. Simion, "Calculul tensiunilor induse in conductele subterane", Research report (in romanian), CEEX - ICEMECOS X2C37, july 2007.

[7] D.D. Micu, E. Simion, D. Micu, A. Ceclan, L. Cret, "Numerical algorithm for the accurate evaluation of the induced voltages in a pipeline", $6^{\text {th }}$ International Conference on Computational Electromagnetics, Aachen, Germany, pp. 230232, April 4-6, 2006.

[8] D.D. Micu, D. Micu, E. Simion, L. Cret, "Numerical computation of the induced voltages in a pipeline using special interpolation functions", International Conference on Electrical Machines, Drives and Power Systems, ELMA Sofia, Bulgaria, Sept. 15-16, pp. 327-329, 2005.

[9] A. Tikhonov, et all, "Numerical Methods for the Solution of Ill-Posed Problems", Kluwer Academic Publishers, London, 1995.

[10] D.D. Micu, A. Ceclan, D. Micu, E. Simion, “ Synthesis Method of an Inductive Sensor using Tikhonov Regularization Procedure", International Journal of Applied Electromagnetics and Mechanics, Vol. 26, Nos 3,4, 2007, pp. 243 - 250.

[11] J. Epperson, "An Introduction to Numerical Methods and Analysis", John Wiley \& Sons Inc., New-York, U.S.A, 2001.

[12] J. Liu, F. Dawalibi, Y. Yang, "Determination of Rail Conductor Characteristics for Electromagnetic Interference Studies", IEEE, 2002, pp. $382-386$. 